\title{
Using multiple reference genomes to identify and resolve annotation inconsistencies
}

\author{
Patrick J. Monnahann 1,2,3, Jean-Michel Michno ${ }^{1}$, Christine O'Connor ${ }^{1,2}$, Alex B. Brohammer ${ }^{1}$, Nathan M. Springer ${ }^{3}$,
} Suzanne E. McGaugh ${ }^{2}$ and Candice N. Hirsch ${ }^{1 *}$ (D)

\begin{abstract}
Background: Advances in sequencing technologies have led to the release of reference genomes and annotations for multiple individuals within more well-studied systems. While each of these new genome assemblies shares significant portions of synteny between each other, the annotated structure of gene models within these regions can differ. Of particular concern are split-gene misannotations, in which a single gene is incorrectly annotated as two distinct genes or two genes are incorrectly annotated as a single gene. These misannotations can have major impacts on functional prediction, estimates of expression, and many downstream analyses.

Results: We developed a high-throughput method based on pairwise comparisons of annotations that detect potential split-gene misannotations and quantifies support for whether the genes should be merged into a single gene model. We demonstrated the utility of our method using gene annotations of three reference genomes from maize (B73, PH207, and W22), a difficult system from an annotation perspective due to the size and complexity of the genome. On average, we found several hundred of these potential split-gene misannotations in each pairwise comparison, corresponding to 3-5\% of gene models across annotations. To determine which state (i.e. one gene or multiple genes) is biologically supported, we utilized RNAseq data from 10 tissues throughout development along with a novel metric and simulation framework. The methods we have developed require minimal human interaction and can be applied to future assemblies to aid in annotation efforts.

Conclusions: Split-gene misannotations occur at appreciable frequency in maize annotations. We have developed a method to easily identify and correct these misannotations. Importantly, this method is generic in that it can utilize any type of short-read expression data. Failure to account for split-gene misannotations has serious consequences for biological inference, particularly for expression-based analyses.
\end{abstract}

Keywords: Annotation, Genome assembly, Maize, Split-gene

\section{Introduction}

The annotation of a genome is a useful resource in many modern sequencing endeavors. It provides the initial link connecting mapping studies to functional impact, and

\footnotetext{
* Correspondence: cnhirsch@umn.edu

'Department of Agronomy and Plant Genetics, University of Minnesota, St. Paul, MN 55108, USA

Full list of author information is available at the end of the article
}

defines the context in which much of our genomic inference takes place. Modern software/pipelines [1] greatly facilitated production of de novo annotations for a large number of species, and multiple independent genome assemblies and annotations have been produced for more well-studied species [2-5].

Despite the importance of developing high quality annotations, and the exponential increase in annotated 
sequences over time that have come from assembly of many new genomes, the annotation process remains notoriously error-prone $[1,6,7]$. Annotation pipelines attempt to integrate multiple data types, such as RNAseq, orthologous protein sequences, ESTs, as well as ab initio predictions from the genome sequence itself. In addition to the complexity of the data, the challenge is heightened by the complexity (and scale) of the underlying biological processes. Expression and maturation of transcripts and proteins is a highly dynamic process that varies over time as well as across different tissues, making it hard to differentiate between functional and intermediate forms. Furthermore, biological errors such as transcriptional read-through, as well as chimeric transcripts, provide conflicting evidence to the true underlying gene(s).

Research communities recognize the value of manual curation in the improvement of annotations and have encouraged input from community members [8, 9]. Manual curation of gene annotations often comes from individual community members interested in a particular gene or gene family, relying on their detailed knowledge and data to identify and correct errors in a gene model. Depending on the community size and resource availability to a given study system, the extent to which this manual curation occurs and is effectively absorbed and corrected in future annotations is variable. Bioinformaticians can facilitate this process by developing automated algorithms that flag potential errors for subsequent manual curation.

The presence of multiple de novo genome assemblies and de novo annotations for a single species or multiple closely related species provides a useful dataset for such algorithms. By identifying the co-linear regions within each reference and linking the homologous genes across the annotations, researchers can discover discrepancies between gene models in the different genome assemblies. One particularly insidious discrepancy is when two distinct gene models in one annotation correspond to non-overlapping parts of a single, merged gene in the alternative annotation, commonly known as split-gene misannotation [10]. These can have major impacts on functional predictions, estimates of expression, as well as downstream analyses. Here, we present a method to compare annotations and automatically detect potential split-gene misannotations, and subsequently determine which gene model (merged vs split) is likely correct, using transcript abundance estimates from short-read sequence data. Expression data from multiple tissues is standard input for most annotation pipelines [1, 11-16], so in most cases, it should exist by virtue of having produced an annotation. This generic method accommodates all standard RNAseq libraries, including single-end and non-stranded preparations.
The difficulty of the annotation process, and thus the prevalence of errors, vary greatly across study systems due to factors such as current and/or ancient polyploidy, transposable element (TE) content, and gene density throughout the genome. Maize is a good case system in which to test our misannotation detection method as it is an ancient polyploid with high TE content including TEs that are in close proximity to gene models. We analyzed de novo annotations from three maize genome assemblies, including W22 [12], B73 [13, 17], and PH207 [11]. Using our pipeline, we identified hundreds of instances where multiple genes corresponded to a single gene in an alternate annotation and determined the most likely annotation. We further demonstrate the biological misinterpretations that can result from these split-gene misannotations.

\section{Results \\ Split-gene Misannotation detection and classification pipeline overview}

Our pipeline proceeds in two major steps: 1.) identification of potential split-gene misannotations (i.e. splitgene candidates) based on pairwise alignments (Fig. 1; Syntenic Homology Pipeline in Methods) followed by 2.) determination of the supported gene model using shortread expression data (Fig. 2; Split-gene classification in Methods). The output of the first step, which is based on a sequential alignment procedure using nucmer followed by reciprocal BLAST, is a key that labels the genes that have a one-to-one homologous relationship across the annotations along with the genes that have a one-to-many homologous relationship (a single gene in one annotation corresponds to multiple genes in the alternative annotation). The one-to-many genes will contain both tandem duplicates as well as split-merge candidates (Fig. 1a). These two classes of one-to-many genes are distinguished by the proportionate overlap of the BLAST query genes with respect to the total aligned space of the subject gene (Fig. 1b). The split-gene candidates are carried forward to the second 'classification' step in the pipeline.

Our classification method is based on the expectation that the difference in expression across the split genes should be greater if split (multiple) gene annotation is correct than if the merged (single) gene annotation is correct. To evaluate this degree of difference in expression patterns across the split genes, we developed the M2f ('Mean 2-fold split-gene expression difference') metric (Fig. 2a-b). Simulated, empirical null distributions (Fig. 2c-d) are then used to determine significance thresholds for the M2f metric, based on if the value is lesser or greater than expected by chance. In other words, are the expression differences across the split- 
A.)

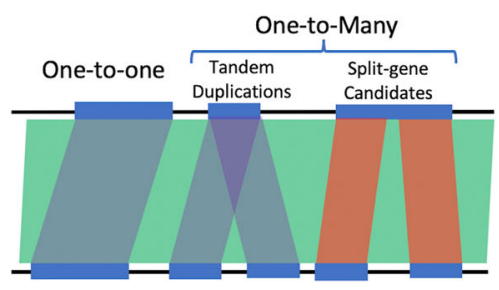

B.)

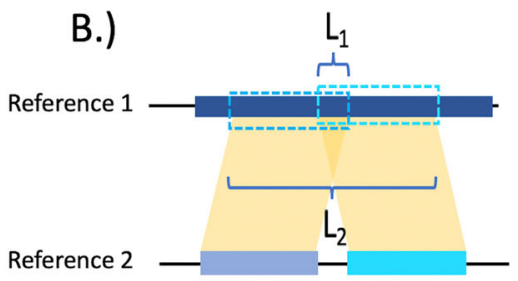

C.)

\begin{tabular}{cccc} 
& B73 x PH207 & B73 x W22 & PH207 x W22 \\
\cline { 2 - 4 } One-to-one & 21307 & 24109 & 21208 \\
One-to-many & 1826 & 1267 & 2214 \\
Opposite strands & 704 & 171 & 752 \\
Tandem Duplications & 227 & 296 & 227 \\
Split-gene candidates & 895 & 800 & 1235 \\
Testable candidates & 687 & 558 & 1063
\end{tabular}

D.)

\begin{tabular}{cccc} 
& Unique & Corroborated & C / U \\
\cline { 2 - 4 } B73 - Split & 422 & 42 & 0.097 \\
B73 - Merged & 583 & 61 & 0.104 \\
PH207 - Split & 525 & 302 & 0.575 \\
PH207 - Merged & 368 & 102 & 0.277 \\
W22 - Split & 400 & 107 & 0.267 \\
W22 - Merged & 662 & 155 & 0.234
\end{tabular}

Fig. 1 Identifying syntenic homologs and isolating split-gene candidates. a Homology classifications from syntenic homology pipeline. b Schematic for calculation of tandem duplicate percentage. We require the ratio of $L 1$ to $L 2$ to be $<0.1$ (i.e. the proportionate overlap of the BLAST query genes with respect to the total aligned space of the subject gene). c Summary of homology classifications and split-gene candidate filtration. A 'Testable candidate' is one in which all of the genes involved are expressed. $\mathbf{d}$ Corroboration of testable candidates. E.g. 43 'Corroborated' split-gene candidates in the B73 annotation ('B73 - Split') were simultaneously identified as a single gene in W22 and PH207, while there were 61 genes in B73 that corresponded to multiple genes in both PH207 and W22 ('B73 - Merged'), and the 438 'Unique' split-gene candidates in B73 were identified as a single gene in W22 or PH207

genes consistent with an underlying biological reality of a single gene or multiple, distinct genes?

To demonstrate the utility of this identification and classification method, we analyzed three maize reference genome assemblies that each of been independently annotated. The annotations under consideration represent different stages of development as well as different types and amounts of validating data. The annotation for B73 is currently in its fourth version, whereas W22 and PH207 are in their second and first version, respectively. Annotation of B73 was based on five evidence types, including long- (PacBio IsoSeq) and short-read RNAseq, optical mapping, full length cDNAs (from BACs), and orthologous protein sequences [17]. The IsoSeq expression data from B73 was also utilized for annotation of W22 as well as short read data and optical mapping
A.)

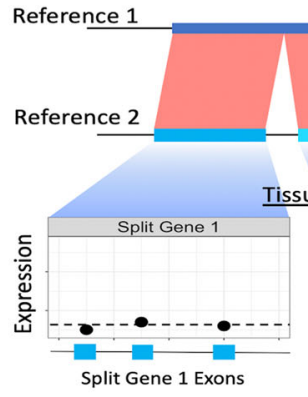

C.)

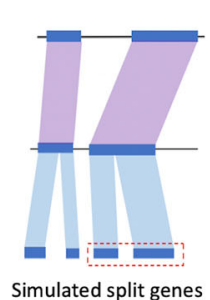

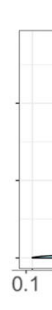

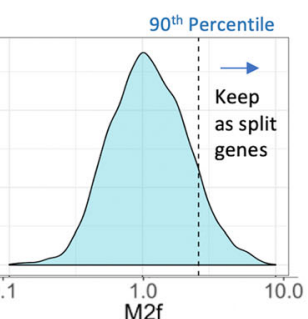

D.)

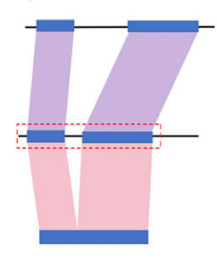

B.)
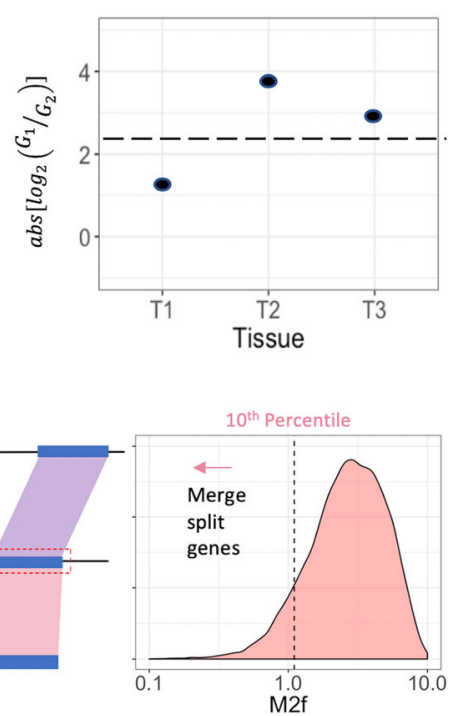

Fig. 2 M2f approach for determining correct gene model(s) for split-gene candidates. a Calculating average normalized expression across exons within a tissue for a pair of split-gene genes. $\mathbf{b}$ M2f calculation. The absolute $\log _{2}$-fold change in average expression (from $\mathbf{a}$ ) across the splitgenes is averaged across tissues. Higher values reflect large expression differences across split-genes. c Simulating the M2f distribution under the null hypothesis that split-gene expression differences come from a single underlying gene. Observed M2f values greater than the 90th percentile of this null distribution are unlikely to result if the single gene annotation is correct. $\mathbf{d}$ Simulating the M2f distribution under the null hypothesis that split-gene expression differences come from separate, adjacent genes 
specific to W22 [12]. The PH207 annotation included only standard short-read RNAseq data from PH207 [11]. All annotations were produced using the MAKER-P pipeline [18] (with a modification for long-read expression data for B73 and W22) and contain approximately the same number of genes $(\sim 40 \mathrm{k})$. Due to the lesser data used for the genome and annotation of PH207, the completeness and accuracy are predictably lower for PH207.

\section{Identification of maize candidate genes}

Alignments generated using nucmer covered a large portion of the genome with the greatest total alignment length between B73 and W22 (1.07 Gb; 46\%). Pairwise alignments with $\mathrm{PH} 207$ covered a much lower ( 37\%) proportion of the genome, regardless of whether it was aligned to B73 or W22. Furthermore, the alignments with $\mathrm{PH} 207$ were broken up into many smaller aligned regions ( $60 \%$ of the average length in B73 x W22; Additional file 1: Table S1). From the syntenic homology pipeline (Fig. 1a) for each pairwise comparison, we found $>20 \mathrm{k}$ one-to-one homologs (with the greatest number identified in the B73 x W22 comparison, likely due to the shared IsoSeq data). We also found 1.2-2.3 thousand instances of one-to-many homology across the pairwise comparisons (with the greatest numbers identified for comparisons involving PH207; Fig. 1c; list of one-to-one and one-to-many homologous genes in Additional files 2 and 3, respectively). Of these one-to-many instances, the most common source were cases with multiple genes in $\mathrm{PH} 207$ that corresponded to a single gene in either B73 or W22. However, in 37\% (comparison to B73) and $44 \%$ (comparison to W22) of these instances, the split PH207 genes were on opposite strands, and often overlapping (Additional file 1: Table S2), perhaps indicative of overannotation of antisense transcription events in PH207. Such opposite and overlapping split-genes were also observed in B73 and W22, but to a much lesser extent (Additional file 1: Table S2).

After filtering the remaining one-to-many candidates to remove possible tandem duplications and retain only expressed genes, there remained substantially more split-gene candidates ('Corroborated' + 'Unique' $=507+$ 307 = 814; Fig. 1d) in PH207 versus B73 (481) and W22 (525). Furthermore, the number of split-gene candidates in $\mathrm{PH} 207$ that were found to correspond to a single gene in both B73 and W22 (i.e. they were 'Corroborated'; Fig. 1d) is much higher than the 'Corroborated' B73 and W22 split-gene candidates combined. This is again concordant with comparatively less data used for the PH207 annotation, where for example, a lowly-expressed gene in $\mathrm{PH} 207$ might lack the coverage necessary to generate a full-length assembled transcript, resulting in annotation of multiple genes instead of the single, true gene.
Considering these split-genes along with the merged genes to which they corresponded, our analysis concerns 1275,1383 , and 2125 genes in the W22, B73, and PH207 annotations, respectively, corresponding to roughly 3$5 \%$ of all genes contained in these annotations. Attributes of these genes tend to be comparable in many regards to the one-to-one homologous genes, except that they are usually nearer to neighboring genes and show more tissue specific expression (Additional file 1: Figure S1).

\section{Classification of maize Split-merge candidate genes using the M2f metric}

For each of the split-gene candidates identified with the syntenic homology pipeline (Fig. 1a), we sought to determine the gene model(s) with greatest support (i.e., should the split-genes remain split or be merged into a single gene?) using our M2f metric. The observed distributions of M2f for the split-gene candidates from each annotation are presented in Fig. 3a, along with the threshold values (dotted lines) from the simulated, null distributions. We observed clear differences in the overall distributions of the M2f metric across the different genotypes (Fig. 3a, Table 1), which leads predictably to differences in the number of split-gene candidates classified as either merged (i.e., the annotation in which the split-genes were annotated as a single gene is supported) or split (i.e., the separate, split-gene annotation is supported) (Fig. 3a-b). The list of split-gene candidates, along with the supported annotation, are provided in the Additional file 10

The M2f distribution of split-gene candidates in the PH207 annotation (the lowest quality annotation, which make up a majority of the overall split-gene candidates) is shifted left relative to the other annotations (Fig. 3a, Table 1), indicating that many of these are likely misannotations and should be merged as they have been annotated in either W22 and/or B73 (Fig. 3b). Out of the 1129 sets of split-gene candidates in the PH207 annotation that were identified in either the comparison with B73 or W22, we found 505 that should be merged versus only 162 that should remain as separate genes. We were unable to make classification for 462 candidate sets based on the 10th and 90th percentiles of the simulated distributions. We observed the opposite pattern for split-gene candidates in the high-evidence B73 annotation (96 split-genes should be merged, 170 should remain as separate genes despite being merged in PH207 or W22, and 240 were unable to be called), where the separate gene models tended to have higher support based on M2f. The B73 gene model(s) tended to be favored by the M2f metric overall in comparison with either W22 or PH207, in line with B73 having the deepest evidence sources used to develop the annotation. 

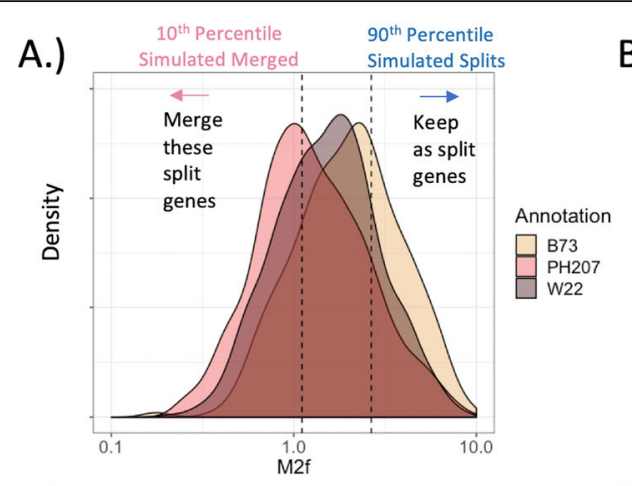

B.)
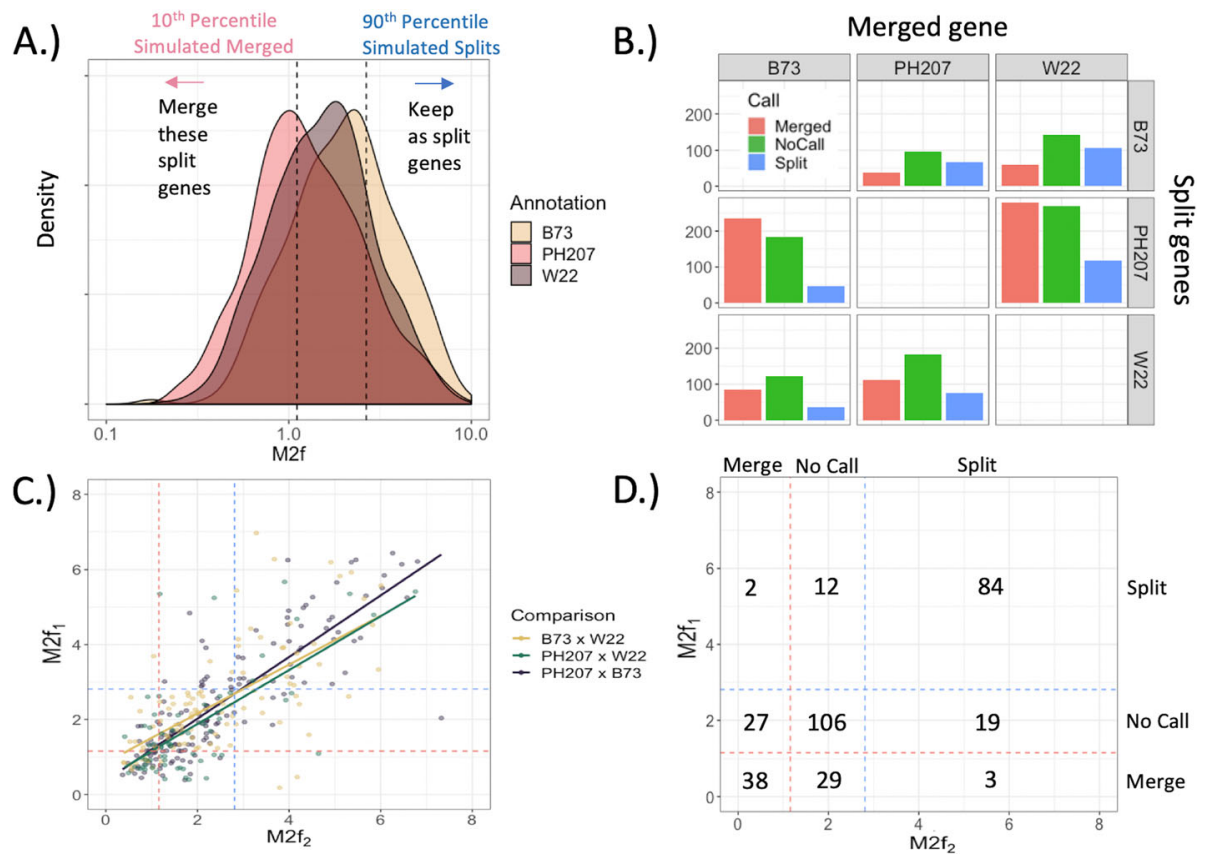

Fig. 3 Results of M2f classification. a Observed M2f distribution across all split-genes detected in each annotation. The dotted lines are the threshold values generated by simulating null distributions in Fig. 2c-d. b Number of split-gene candidates (Multiple genes) classified as to whether the split-genes should be annotated as distinct genes or a single, merged gene for each pairwise comparison of annotations. $\mathbf{c}$ Correlation of M2f values for instances where a single gene from one annotation corresponded to split-gene candidates in both of the alternative annotations ('Corroborated' Merged genes in Fig. 1d). E.g. Each point in the 'B73 x W22' comparison corresponds to a single PH207 gene. X-axis is the M2f value from the B73 split-gene candidate, and $y$-axis is the M2f value from the W22 split-gene candidate. Dotted lines indicate the M2f threshold values in part $\mathbf{a}$. $\mathbf{d}$ Joint distribution of classifications across comparisons in part $\mathbf{c}$

Having multiple pairwise comparisons also allows us to determine the consistency of the M2f metric. We consider instances where a single gene in one annotation corresponded to multiple genes in both of the alternative annotations. This provides two M2f values for this single gene, which should be correlated if M2f is sensitive to the underlying biological truth. In Fig. 3c, we plot this correlation in M2f metrics for each annotation. In this plot, the 'B73 x W22' correlation concerns the single PH207 genes that corresponded to multiple genes in both B73 and W22. We found this correlation is highest when W22 is the annotation with a single gene corresponding to multiple genes in both PH207 and B73 (B73 vs. $\mathrm{PH} 207$ correlation $=0.85)$, followed by $\mathrm{B} 73(\mathrm{PH} 207$ vs. W22 correlation $=0.68)$ and $\mathrm{PH} 207(\mathrm{~B} 73$ vs. W22 correlation $=0.66$ ). While these correlations are

Table 1 Summary of M2f distributions for split-gene candidates in each annotation. $\mathrm{CV}=$ coefficient of variation. $N=$ number of tested candidates

\begin{tabular}{llllll}
\hline Split-genes & Mean & Median & Variance & CV & N \\
\hline B73 & 2.45 & 2.09 & 2.49 & 0.693 & 506 \\
PH207 & 1.64 & 1.2 & 2.07 & 0.88 & 1129 \\
W22 & 2.05 & 1.66 & 2.42 & 0.759 & 614 \\
\hline
\end{tabular}

imperfect, they rarely lead to conflicting classifications (Fig. 3d) and, typically, the M2f value trends in the same direction even if the gene model does not pass the null distribution thresholds. Of the 320 instances where a single gene corresponded to two or more split-genes in both of the alternate annotations, only five $(1.56 \%)$ are in conflict (i.e. M2f supports merging the split-genes for one of the alternative annotations, while the other alternative annotation suggests the genes should be kept separate, or vice versa; Fig. 3d).

To further test the robustness and validity of our approach we investigated a number of potential confounding factors (Additional file 1: Figures S2-4) that could impact classification of genes based on the M2f metric. First, we examined if genes that produce multiple isoforms have inflated M2f values. We compared the M2f distributions for B73 genes with multiple isoforms versus single isoforms (Additional file 1: Figure S2) and found a slight inflation of M2f values for genes with multiple isoforms (Median M2f of 1.41 vs 1.59 for single and multiisoform genes, respectively, within the split-gene candidates). Although this bias is slight, it serves to emphasize the role of the simulations in protecting against potential artifacts. As long as the simulated data is representative of our split-gene candidates (multiple isoform genes, in 
this case, are not over-represented in our candidates), the simulated null distribution will include this M2f inflation, thus protecting against misclassification due to this artifact. Notably, in our study, multi-isoform genes within our B73 candidates are less frequent in the empirical data (0.54) than to either the simulated split genes (0.64) or the simulated merged genes (0.59). We also explored the impact of exon number on our M2f metric and found that there is little impact of exon number on the distribution of M2f values (Additional file 1: Figure S3). Finally, we explored the impact of using annotations from the different genome assemblies to set the thresholds for setting the 10th and 90th percentiles, and found the thresholds were highly similar across the genomes (Additional file 1: Figure S4).

\section{Features of classified maize genes}

We explored features of the classified genes to determine if there were common features that could be informative in improving future automated annotation efforts. Genes that were originally annotated as a single/ merged gene model but were determined to be split based on the M2f metric tended to be longer (Fig. 4b) and have more exons (Additional file 1: Figure S6a). Merged gene models supported by our M2f metric (MS = merged supported) were longer than the misannotated, merged genes (MNS = merged not supported); yet,
MS genes have comparatively fewer exons than MNS genes (Additional file 1: Figure S6a,c). The long, exonsparse MS genes may be more likely to be missing reads spanning particular exon-exon junctions and, thus, be more prone to being misannotated as multiple genes (particularly when relying on short-read RNAseq data).

Generally, the split-gene candidates (including genes originally annotated as split, along with their merged counterparts in the alternate annotations), tend to be closer to other genes as compared to the genes with one-to-one homology across all three annotations (median distance of $3.6 \mathrm{~kb}$ versus $4.1 \mathrm{~kb}$ ). This suggests that gene dense regions may be more prone to split-gene misannotations, and that these misannotations may be more frequent in species with smaller, gene-dense genomes. Looking within the split-gene candidates (all categories except for 'One-to-one' in Fig. 4), we found that when split gene annotation is supported, the components of the unsupported merged gene tend to be closer together. This suggests that the distance between these components contributed to the misannotation as a merged gene, potentially through a mechanisms like transcriptional read through of proximate genes. We observed the opposite trend in the PH207 annotation, but only for the split-genes in $\mathrm{PH} 207$ that corresponded to a single gene in W22 (split not supported (SNS) distance = $3.6 \mathrm{~kb}$; SS distance $=5.3 \mathrm{~kb}$ ).

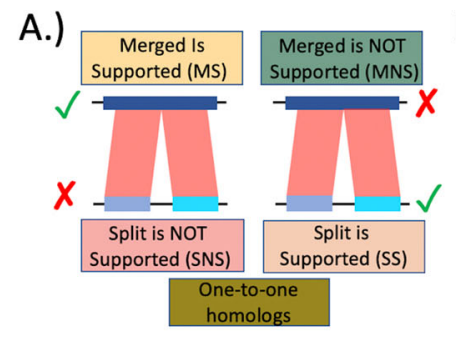

C.)

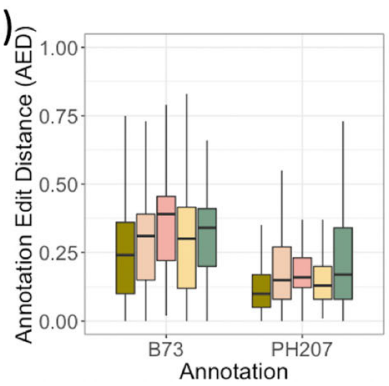

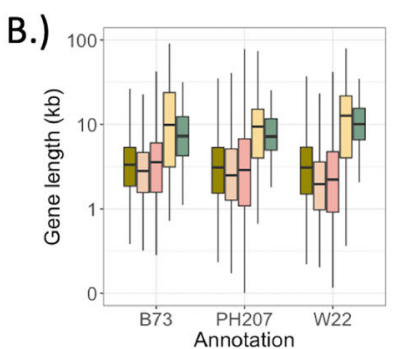

D.)

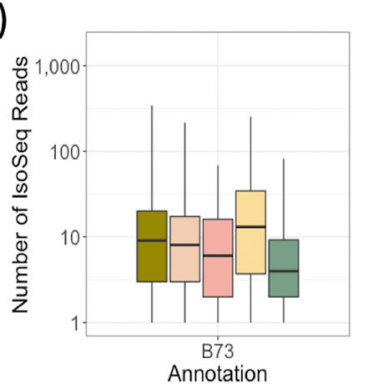

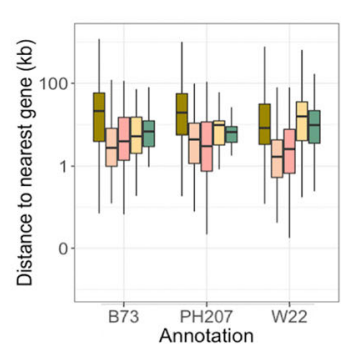

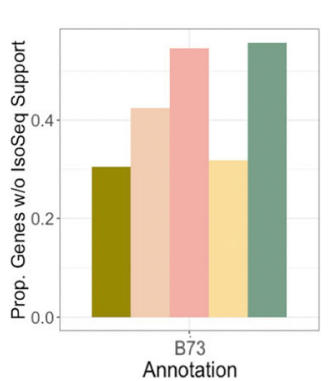

Fig. 4 Features of one-to-one genes as well as split-gene candidates. a Split-gene candidates are classified based on whether they were initially annotated as split or merged for a given genotype followed by the classification based on the M2f method. E.g. The 'SS' box for the B73 genotype are instances where multiple genes in B73 corresponded to a single gene in either PH207 or W22, and the multiple (split) genes of B73 were determined to be the correct annotation. Outliers were removed on all plots. b Length and Distance between genes. c AED calculated from MAKER-P for the B73 and PH207 annotations. For B73, multiple isoforms were annotated, and we took the max AED across all isoforms for a given gene model. $\mathbf{d}$ Number of IsoSeq cDNAs for genes in each category. Genes with no IsoSeq support were excluded and shown separately as a proportion on the right. IsoSeq cDNAs were filtered for mapping quality $(\mathrm{MQ})>20$ and for coverage of at least $75 \%$ of the longest transcript sequence 
We also investigated whether expression differed between supported and unsupported annotations. Overall, expression abundance did not markedly differ from that seen in the one-to-one genes (Additional file 1: Figure S6a). One slight exception is for the genes that were incorrectly annotated as a single, merged gene (MNS), where there is a higher density of high expression for these 'genes'. Increased expression of one or multiple proximate, distinct genes may increase the likelihood of producing chimeric transcripts (e.g. via transcriptional read through), thus promoting incorrect annotation as a single, merged gene. Tissue-specificity of expression differed markedly between classification categories (Additional file 1: Figure S5a,b), particularly for the highly tissuespecific genes (Additional file 1: Figure S5b). We found that split-gene annotations (both split supported (SS) and SNS) were more likely to result when expression of one of the genes was highly tissuespecific, whereas merged gene annotations (both MS and MNS) occurred more often when expression was less tissue-specific. Interestingly, within each of these categories, the subset of supported annotations (as determined by our M2f metric) tended to be more tissue-specific than the non-supported annotations (Additional file 1: Figure S5b).

The annotation edit distance (AED) is a common annotation quality metric that can be used to summarize the differences between an annotated gene model and the supporting evidence [19]. We found that the AED reported by MAKER-P for the B73 and PH207 annotation is consistently higher for split-gene candidates as compared to the one-to-one homologs (Fig. 4c), indicating lower quality of these gene models, generally. Notably, the AED of nonsupported annotations (SNS and MNS) is higher than the supported annotations (SS and MS). However, the AED distributions of supported and nonsupported split-gene annotations are largely overlapping; thus, while AED is sensitive to split-gene misannotation, it cannot be used to robustly identify incorrectly merged or split gene models.

We found that nonsupported annotations in B73 have lower or no IsoSeq coverage as compared to supported annotated gene models (Fig. 4d). Both of the nonsupported annotation categories (SNS and MNS) have the highest proportion of genes with no long-read support ( $\mathrm{SNS}=0.54$ and $\mathrm{MNS}=0.58$ versus $\mathrm{SS}=0.42$ and $\mathrm{MS}=0.32$ ). When we consider only the genes that have long-read support, there tend to be fewer supporting reads for the nonsupported annotation categories, particularly when B73 has a nonsupported, merged gene that M2f suggests should be split (Median number of IsoSeq cDNAs for $\mathrm{MNS}=4$ and $\mathrm{SNS}=7$ versus $\mathrm{MS}=11$ and $\mathrm{SS}=8$ ).

\section{Consequences of Split-gene Misannotations on biological findings}

We explored the consequences of split-gene misannotations for biological inference that rely heavily on the annotation, namely expression-based analyses. Comparing across genotypes, we found that genes that are one-toone homologs show a much tighter correlation in normalized expression $(r=0.92)$ than the correlation between supported split-genes and their corresponding (nonsupported) single, merged gene ( $r=0.43$; Fig. 5a; SS category in Fig. 4). If two distinct genes are incorrectly annotated as a single gene, the estimated expression for the single gene will be an average of the expression of the two loci. Unless the two loci happen to be expressed similarly, this average will likely be more dissimilar from either of the two distinct genes than if we were to compare expression with the true homologs (i.e. if the misannotated merged gene was correctly annotated as two distinct genes). The dissimilarity may be further amplified by normalization procedures that scale read counts by the length of the feature over which expression is being measured. For an equivalent number of reads, the longer, merged gene model will have lower normalized expression. On the other hand, when the single, merged gene was supported, we found a very tight correlation between the expression of this gene and the corresponding (non-supported) split-genes $(r=0.99$; Additional file 1: Figure S7).

Poor estimations of transcript abundance for splitgene candidates presumably will have consequences on inference of differential expression as well as differential exon usage. For example, the two PH207 genes in Fig. $5 \mathrm{~b}$ are differentially expressed albeit in opposite directions across the immature ear and anthers, yet these differences cancel out when we test for differential expression of the single, merged gene as annotated in W22 (Fig. 5b). Similarly, Fig. 5c illustrates improper inference of differential exon usage of the left-most exon in two of the tissues, when in fact, this exon is a distinct (and differentially expressed) single-exon gene according to our results. Across all of the non-supported merged genes, there is an abundance of differential exon usage as compared to the supported merged genes (Fig. 5d), suggesting that unsupported merged gene models lead to false inference of differential exon usage. We also observed this trend for the DESeq2 analysis, albeit to a lesser degree (Additional file 1: Figure S8). A much higher proportion of exons are inferred to be differentially used across tissues for these non-supported gene models, which is expected when the non-supported merged gene is composed of two or more multi-exon genes (Additional file 1: Figure S9). Therefore, these types of misannotations are highly predisposed for misinference of underlying biological processes. 
A.)

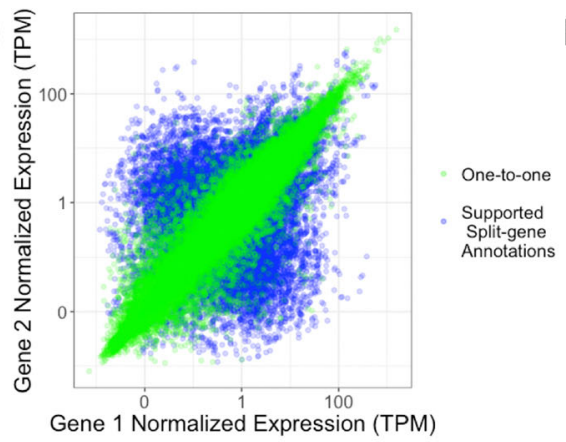

C.)

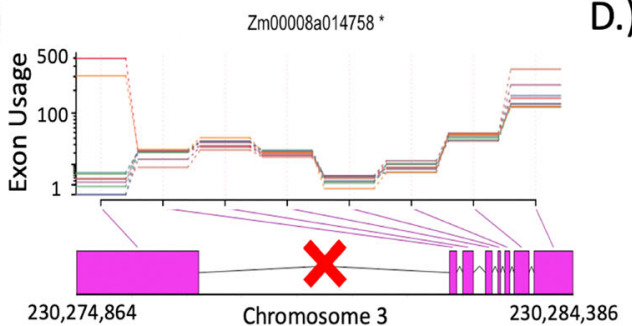

B.)

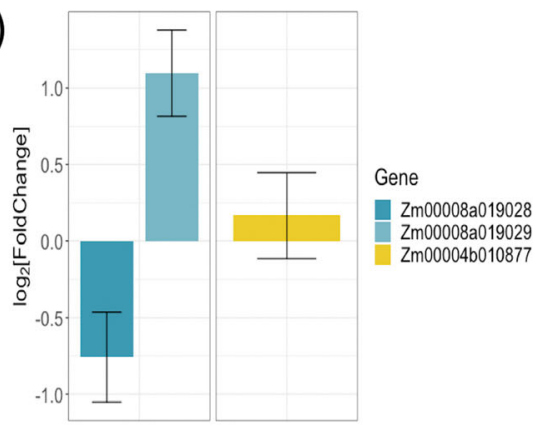

D.)

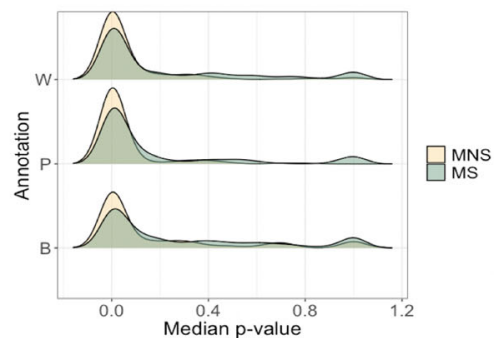

Fig. 5 Consequences of split-gene misannotations. a Comparing expression estimates across homologs. For the correct split-gene annotations (Split Supported (SS) in Fig. 4), expression of each split-gene is compared to the one expression value from the single gene to which they corresponded. b Exemplar of differential expression misinference when two distinct genes are incorrectly annotated as one. Expression differences (between immature ear and anther) of component genes cancel out resulting in no differential expression for the single rightmost gene. c Example of misinference for differential exon usage. Incorrect annotation as a single gene in PH207 should be two genes (split at location demarcated with the red X) as annotated in W22. Colored lines indicate separate tissues. $\mathbf{d}$ Median $p$-value across the per-exon tests of differential exon usage for each gene. Inflation of low p-values is observed when distinct genes are incorrectly treated as a single, merged gene (Merged is not supported (MNS) in Fig. 4)

\section{Discussion}

Accurate gene models are of paramount importance in the era of genomics. While the bioinformatics community continues to develop and improve tools for the prediction of gene models (i.e. annotation), the burden of verifying and, if necessary, correcting these predictions is largely spread across the individuals invested in researching the particular organism. Bioinformaticians can do more to facilitate this process by developing methods that flag/correct misannotated genes, preferably without requiring the generation of additional data. We have described a comparative approach to identify potential split-gene misannotations across annotations of individuals within a species or closely related species, and a method to infer the correct annotation using preexisting RNAseq data.

Though our approach is based on short-read RNAseq data, the utility of long-read expression data is clear in our results. PH207, which was the only annotation that did not utilize long-read data, exhibited substantially more split-gene misannotations than W22 and B73 combined. A single long read can capture all of the exonexon junctions, whereas observations on one or more junctions are more likely to be missing with short-read sequencing due to random variation in sequencing coverage. In line with this, we found split-gene misannotations are more often associated with lowlyexpressed and/or tissue-specific genes.

We have, however, shown that even annotations that are based on long-read data will still contain split-gene misannotations. These misannotations are not due to the long-reads per se; they more likely result whenever long-read data is unavailable for a particular gene and short-reads are sparse (e.g. lowly expressed genes). Our method capitalizes on the fact that these same genes may be more highly expressed in other genotypes, thus providing more complete evidence of the underlying gene model. The more salient issue with long-reads (and short-reads, for that matter) is the potential for aberrant transcriptional readthrough events that encourage improper merging of adjacent gene models [20]. Fortunately, such events ought to be detected by our method, as these merged genes will more likely show highly inconsistent expression patterns.

In its current implementation, our method will not detect all instances of split-gene misannotations. Thus, we may underestimate the abundance of split-gene misannotations. The most obvious cause of non-detection would be if the gene(s) were consistently misannotated across all of the annotations being compared, in which case we would identify these genes as one-to-one homologs. However, by increasing the number of independent 
annotations considered, we should increase the odds that at least one annotation possessed the correct gene model. Additionally, our method will not identify split/ merge candidates in which the gene(s) are only annotated in one of the genomes being analyzed. In the cases, additional information such as full-length cDNA sequences would be required to identify the split candidates. We also are only considering split-gene candidates where both of the split genes are expressed. Our attempts to handle the 0-expression genes introduced clear artifacts in our M2f metric, though an alternative or modified metric could possibly accommodate these scenarios. Lastly, we cannot strictly discriminate between truly split genes and certain scenarios of a single gene with multiple isoforms. Our simulation framework will partly protect against M2f inflation from multiple isoforms, since multiple isoform genes were wellrepresented in the simulated split or merged genes. However, a multi-isoform gene in which the predominant isoform is simply a truncated version of the longest isoform may still result in false positives via our approach. For these reasons, we view our method as a high-throughput means of flagging potential misannotations and suggesting the correct gene model, in order to facilitate the manual curation process of the larger community $[8,9]$.

\section{Conclusions}

In summary, as additional de novo genome assemblies and annotations are produced, the greater the opportunity to identify and correct errors and inconsistencies. We have described a method to facilitate this process for split-gene misannotations, which we have demonstrated can strongly bias a range of biological estimates. Given that the required input (RNAseq) is readily available by virtue of having produced an annotation, our method could be integrated as a standard part of the annotation process for systems in which annotations already exist for other genotypes or individuals. Accrual of such tools are an important step towards developing accurate and consistent genome annotations, a foundational resource in the age of genomics.

\section{Methods}

\section{Maize datasets}

We focused on three maize genome assemblies and corresponding annotations for this study: B73 (v4; AGPv4, $\mathrm{ftp} / /$ ftp.ncbi.nlm.nih.gov/genomes/genbank/plant/Zea mays/latest_assembly_versions/GCA_000005005.6_B73_ RefGen_v4) [13, 17], W22 (v2, ftp://ftp.ncbi.nlm.nih.gov/ genomes/genbank/plant/Zea_mays/latest_assembly_versions/GCA_001644905.2_Zm-W22-REFERENCENRGENE-2.0) [12], and PH207 (v1, https://doi.org/10. 5061/dryad.8vj84) [11]. We filtered annotations for a single gene with multiple transcripts by filtering for the longest coding sequence (CDS). We then converted these representative transcripts from GFF to fasta format and created a BLAST database [21] for each reference.

For each of the three genotypes, we collected tissue from ten spatiotemporally diverse tissues including: (1) primary root six days after planting, (2) shoot and coleoptile six days after planting, (3) internode at the Vegetative 11 developmental stage, (4) middle of the 10th leaf at the Vegetative 11 developmental stage, (5) middle of the leaf from the ear bearing node at 30 days after pollination, (6) meiotic tassel at the Vegetative 18 developmental stage, (7) immature ear at the Vegetative 18 developmental stage, (8) anthers at the Reproductive 1 developmental stage, (9) endosperm at 16 days after pollination, and (10) embryo at 16 days after pollination. Tissues (1) and (2) were collected from greenhouse grown plants that were planted in Metro-Mix300 (Sun Gro Horticulture) with no additional fertilizer and grown at $27 \mathrm{C} / 24 \mathrm{C}$ day/night and $16 \mathrm{~h} / 8 \mathrm{~h}$ light/dark. Plants were grown at the University of Minnesota Plant Growth Facilities in St. Paul, MN in June of 2015. The remaining tissues were collected from field grown plants that were planted at approximately 52,000 plants per hectare with 30-in. row spacing and grown under standard corn growth conditions. Seeds for field grown plants were planted at the University of Minnesota Agricultural Experiment Station located in St. Paul, MN in May 2015. For tissues (1) through (4) all biological replicates for all genoyptes were collected on a single day. For the remaining tissues the two biological replicates for a given genotype were collected on the same date. However, the date of collection between genotypes was variable and determined by the time to reach the specified developmental stage. Tissue sampling for each of the tissues was conducted using previously described methods $[22,23]$. These tissues were chosen to broadly capture variation in the maize transcriptome based on the maize B73 gene atlas [22]. We collected two biological replicates per genotype/tissue combination and standard, non-stranded RNAseq libraries were prepared for each tissue sample using the Illumina TruSeq library preparation protocol-replicate.

Libraries were sequenced on an Illumina HiSeq 2500, using $50 \mathrm{bp} \mathrm{SE}$ reads (avg. number of reads $=30.5$ million; see Additional file 1: Table S3 for total reads per sample). We checked the quality of each file with FASTQC (version 0.11.7) [24] and subsequently performed adapter- and quality-trimming with cutadapt (version 1.16; quality threshold of 20 and minimum retained length of $30 \mathrm{bp}$ ) [25]. We used STAR (version 020201 [26];) to align RNAseq reads to each of the reference genomes on a per-exon basis, allowing for $50 \mathrm{bp}$ of overhanging sequence on either side of the putative 
splice junctions (--sjdbOverhang 50). We sorted, indexed, and filtered (MQ $>2)$ BAM files with samtools (version 1.6 [27];). To count RNAseq reads for each exon, we used HTseq (version 0.10.0) [28] with the stranded option set to 'no' and a minimum quality of 0 (since BAM files were pre-filtered). For each exon, we calculated normalized expression as the number of transcripts per million (TPM), which was chosen based on its ability to compare across libraries [29]. We filtered out any exons less than $50 \mathrm{bp}$ in length as this will influence our ability to map reads to these exons with our 50 bp reads.

Expression counts are available in the supplemental material (Additional files 4, 5 and 6) Scripts used to prepare or generate these materials are available at https://github. com/HirschLabUMN/Split_genes/tree/master/Per_Transcript_Exon_Pipeline.

\section{Syntenic homology pipeline}

Identifying syntenic homologs across each annotation was done in two steps, which included identifying large blocks of synteny between the genomes and comparing specific BLAST searches within those large blocks (Fig. 1a). For the first step, we used nucmer (version 3.1) for each pairwise combination of genomes [30], requiring anchor matches to be unique in both reference and query ('--mum' flag) as well as a minimum cluster length (-c) of $1000 \mathrm{bp}$. We used default settings for the remaining options. We ran the delta-filter utility within the Mummer suite to identify the longest mutually consistent set of matches (-g flag) with a minimum alignment uniqueness of $75 \%$ ( $-\mathrm{u}$ flag). Finally, we used the show-coords utility to convert the output into a table set of coordinates.

For the second step, we began by performing an allby-all BLAST (blastn) using the databases described in the previous section and retaining only the matches with an E-value <1e-4. If there were multiple matches between a given query and subject gene pair, we kept only the single best match based on E-value (length of matching bases was used in case of equivalent E-values). We then filtered matches based on whether the subject and query CDS were within the same nucmer-established syntenic regions $( \pm 500 \mathrm{~kb}$ on each side). Lastly, we searched for instances where proximal subject genes (within five gene models as determined by numeric suffix of gene IDs) matched the same query gene. From this, we classified each query gene as having: 1.) no corresponding gene in the alternative annotation, 2.) a single corresponding gene, or 3.) multiple corresponding genes. We then looked for overlap among the reciprocal BLASTs to confirm syntenic homologous relationships. In the case of a single gene corresponding to multiple genes in one direction, we required that the multiple genes corresponded exclusively to the single gene from the other reference. From the one-to-multiple syntenic homologies, we isolated the potential split-gene misannotations by requiring that the 'multiple' genes are: 1 .) not annotated as overlapping, 2.) on the same strand, 3.) not a tandem duplication based on BLAST (i.e. have less than $10 \%$ overlapping BLAST coordinates calculated as a percentage of total length covered by BLAST hits; L1 / L2 $<0.1$, see Fig. $1 \mathrm{~b}$ for definition of L1 and L2), and 4.) each expressed in our dataset.

\section{Split-gene classification}

Our classification method is based on the expectation that expression across the split genes should be less consistent if the split (multiple) gene annotation is correct than if the merged (single) gene annotation is correct. This implies two requirements: 1.) a metric that distills expression differences across split genes and 2.) critical values from a null distribution that specify values too large or small to be expected by chance.

For each gene we first calculated normalized expression (TPM) for every sequenced tissue (i.e. library) by averaging across exons. Genes with an average transcript per exon less than 0.01 were filtered out to remove lowly expressed genes that cannot be accurately resolved (see Additional file 1: Figure S6 for distribution of TPM values). For each split-gene candidate, we then calculated the $\log _{2}$-fold change in expression across all split genes within the set. We took the absolute value of this $\log _{2}$-fold change to erase the dependence on what is arbitrarily chosen as numerator and denominator (if we do not take the absolute values, then the distribution is centered on 0 , as expected if expression is equivalent across split-genes; Additional file 1: Figure S10). If more than two genes corresponded to a single, merged gene, we then averaged across all possible fold-change values to arrive at a single number summarizing expression differences across the split genes within a single tissue. The final metric for the split-gene candidate set is an average (across tissues and biological replicates) of these absolute $\log _{2}$-fold changes, which we term M2f for 'mean twofold expression change across tissues'.

When calculating this value, we subset the data to include only the genotype corresponding to the annotation with the split, or multiple gene models, in order to provide the best representation of the expression patterns used to create the annotation. If there is differential expression or differential exon usage between genotypes, then utilizing expression data from divergent genotypes could generate a false signal for M2f.

Next, we developed a simulation framework to generate empirical null distributions. The first distribution that we simulated was used to identify split-gene candidates whose expression differences are greater than we 
would expect by chance. For this, any split/merge candidate genes were first removed. Then $20 \%$ of the remaining genes across the three genomes $(17,583$ total genes) were randomly selected. These genes were then 'split' in two at a random position. We chose only genes with at least 4 exons for splitting to avoid simulating an overabundance of single-exon genes, though this minimum exon criteria did not have a large effect on the resulting distributions (Additional file 1: Figure S3). We then calculated the M2f value across artificially split pairs to produce a distribution. Candidates with high M2f values relative to this distribution indicate that these genes show larger differences than we would expect if we were to simply took a truly merged gene and treat it as separate genes. We use the 90th percentile of the null distribution as the threshold to classify that split-gene candidates should in fact stay as separate genes.

The second null distribution was created by again first removing split/merged candidates. Then $30 \%$ of the remaining genes across the three genomes were selected without replacement and the adjacent upstream gene was also selected (48,408 total genes). These pairs of genes were artificially merging them into a single gene. We calculate the M2f values for the original adjacent loci and used the 10th percentile of the distribution for all M2f values for the artificially merged distribution as the threshold below which we classified split-gene candidates as merged (i.e. the single, merged gene model is correct). These distributions were similar across annotations of the different genotypes (Additional file 1: Figure S4), with consequently similar values for the 10th percentile (1.12, 1.08, and 1.13 for B73, PH207, and W22, respectively) and 90th percentile (2.66, 2.52, and 2.79 for B73, PH207, and W22, respectively) of the simulated merged and simulated split distributions, respectively. Thus, for each of these percentiles, we used a single value (1.11 for 10th percentile and 2.66 for 90 th percentile) based on pooling the simulated data across the annotations.

Input files are available in Additional files 7, 8 and 9, output file is available in Additional file 10, and code used to prepare output from the syntenic homology pipeline and classify split-gene candidates is available at https://github.com/HirschLabUMN/Split_genes/tree/ master/scripts.

\section{B73 IsoSeq and AED analysis}

The PacBio IsoSeq data for B73 was downloaded from the SRA (BioProject \#: PRJNA10769; SRA Project ID: SRP067440; SRA Sample Numbers: SRR3147022 through SRR3147057) [13]. These FASTQ files contain intact cDNA fragments, which result from running the raw reads through the IsoSeq processing pipeline. For each of the six tissues, there were six FASTQ files corresponding to non-overlapping size ranges of the cDNA fragments. We mapped each FASTQ to the B73 v4 reference genome assembly, using the splice-aware settings in Minimap2 ('-ax splice -uf -C5') [31]. We then combined all BAM files and filtered for cDNAs with a mapping quality $>20$. We calculated coverage of each gene model using BEDtools [32], requiring that the IsoSeq cDNAs covered $75 \%$ of the gene model (according to the longest transcript; Additional file 11).

Annotation Edit Distance values for the B73 (v4) annotation were made available by the Ware lab at: $\mathrm{ftp} / / /$ $\mathrm{ftp}$.gramene.org/pub/gramene/Zea_mays/Jamboree_materials/. AED scores for the $\mathrm{PH} 207$ annotation are provided in Additional file 12.

\section{Differential expression and exon usage analysis}

To investigate the effect of split-gene misannotations on differential expression and differential exon usage, we utilized the programs DESeq2 (version 1.22.2) [33] and DEXseq (version 1.28.3) [34], respectively. For each of these analyses, we were interested in determining whether conflicting biological conclusions would be drawn for one or more of the split-genes as compared to the single, merged gene and whether such conflicts occur at a higher rate for misannotated split-genes. We subsetted the data to include only the genotype that is not involved in the split-gene candidate set to avoid artifacts due to reference mapping bias. For example, if we are investigating two genes from the W22 annotation that corresponded to a single gene in B73, then we would use only expression data from PH207 (mapped to both W22 and B73). If we used expression data from W22 (again, mapped to W22 and B73) and observed conflicting DE inference (e.g. DE for one of the W22 genes, but no DE for the B73 gene), we would be unable to disentangle whether the conflict was due to the misannotation or reference bias.

Since we are only utilizing expression data from a single genotype, we are restricted to testing for differential expression (or exon usage) across tissues. For DESeq2, we summed expression counts (non-normalized) across exons, filtered genes with no expression, and tested for differential expression with default parameters. For DEXseq, we directly used the per-exon expression counts from HTseq, again with default parameters. Our exact implementation of each of these analyses can be found at https://github.com/HirschLabUMN/Split_ genes/blob/master/analysis/SplitGenes.Rmd.

\section{Supplementary information}

Supplementary information accompanies this paper at https://doi.org/10. 1186/s12864-020-6696-8. 
Additional file 1. Supplemental Figures 1-11 and Supplemental Tables 1-3.

Additional file 2. One-to-one homologs identified in the syntenic homology pipeline.

Additional file 3. One-to-many homologs identified in the syntenic homology pipeline. A filtered subset of these entries are the split-gene candidates analyzed in this study.

Additional file 4. Expression matrix for reads mapped to B73. Sample names have format Genotype-Tissue-Rep and correspond to the names as defined in Table S3.

Additional file 5. Expression matrix for reads mapped to W22. Sample names have format Genotype-Tissue-Rep and correspond to the names as defined in Table S3.

Additional file 6. Expression matrix for reads mapped to PH207. Sample names have format Genotype-Tissue-Rep and correspond to the names as defined in Table S3.

Additional file 7. Formatted input containing B73 candidate and simulated split-genes for classification via the M2f_Classify.R script.

Additional file 8. Formatted input containing W22 candidate and simulated split-genes for classification via the M2f_Classify.R script.

Additional file 9. Formatted input containing PH207 candidate and simulated split-genes for classification via the M2f_Classify.R script.

Additional file 10. Supported annotations according to our M2f procedure.

Additional file 11. IsoSeq CDNA count data. See B73 IsoSeq analysis in Methods for procedure used to generate counts.

Additional file 12. Annotation Edit Distance (AED) scores for B73 and PH207 annotated gene models.

\section{Abbreviations}

TE: Transposable element; M2f: Mean 2-fold split-gene expression difference; MS: Merged supported; MNS: Merged not supported; SNS: Split not supported; SS: Split supported; AED: Annotation edit distance; CDS: Coding sequence; TPM: Transcripts per million; MQ: Mapping quality

\section{Acknowledgements}

We thank the lab of Doreen Ware (particularly, Marcela Tello-Ruiz, Josh Stein, and Cristina Fernandez-Marco) at Cold Spring Harbor Laboratory for sharing information on the B73 v4 annotation as well as for organizing the Maize Annotation Jamboree, a workshop which enables community involvement in improving the B73 annotation.

\section{Authors' contributions}

CNH, NMS, SEM conceived the experiment. PJM, JMM, CO, AB analyzed the data. PJM and $\mathrm{CNH}$ wrote the manuscript. All authors reviewed and approved the final manuscript.

\section{Funding}

This work was funded by the National Science Foundation (Grant IOS1546727) and ABB was supported by the DuPont Pioneer Bill Kuhn Honorary Fellowship and the University of Minnesota MnDRIVE Global Food Ventures Graduate Fellowship.

\section{Availability of data and materials}

The raw expression data supporting the conclusions of this article is available in the NCBI SRA. RNAseq data generated for this project is in repository BioProject number PRJNA543878 [URL: https://www.ncbi.nlm.nih.gov/ bioproject/?term=PRJNA543878\&utm_source=gquery\&utm_medium=search]. Specific SRA accession numbers are in Table S3. Available PacBio IsoSeq data for B73 was downloaded from BioProject number PRJNA10769 [URL: https:// www.ncbi.nlm.nih.gov/bioproject/?term=PRJNA10769]. SRA Project ID SRP067440 and SRA Sample Numbers SRR3147022 through SRR3147057 were used for the analysis in this manuscript. The input files, including the expression counts and the real and simulated split-gene candidates, are included as 'Supplementary information'. Code is available at https://github. com/HirschLabUMN/Split_genes/. The script that generated the tables, figures, and numbers is available at https://github.com/HirschLabUMN/Split_ genes/blob/master/analysis/SplitGenes.Rmd

Ethics approval and consent to participate

Not applicable.

\section{Consent for publication}

Not applicable.

\section{Competing interests}

The authors declare that they have no competing interests.

\section{Author details}

${ }^{1}$ Department of Agronomy and Plant Genetics, University of Minnesota, St. Paul, MN 55108, USA. ²Department of Ecology, Evolution, and Behavior, University of Minnesota, St. Paul, MN 55108, USA. ${ }^{3}$ Department of Plant and Microbial Biology, University of Minnesota, St. Paul, MN 55108, USA

Received: 30 May 2019 Accepted: 24 March 2020

Published online: 08 April 2020

\section{References}

1. Yandell M, Ence D. A beginner's guide to eukaryotic genome annotation. Nat Rev Genet. 2012;13(5):329.

2. Tello-Ruiz MK, Naithani S, Stein JC, Gupta P, Campbell M, Olson A, et al. Gramene 2018: unifying comparative genomics and pathway resources for plant research. Nucleic Acids Res. 2017;46(D1):D1181-D9.

3. Berardini TZ, Reiser L, Li D, Mezheritsky Y, Muller R, Strait E, et al. The Arabidopsis information resource: making and mining the "gold standard" annotated reference plant genome. Genesis. 2015;53(8):474-85.

4. Frankish A, Diekhans M, Ferreira A-M, Johnson R, Jungreis I, Loveland J, et al. GENCODE reference annotation for the human and mouse genomes. Nucleic Acids Res. 2018:47(D1):D766-D73.

5. Thurmond J, Goodman JL, Strelets VB, Attrill H, Gramates LS, Marygold SJ, et al. FlyBase 2.0: the next generation. Nucleic Acids Res. 2018;47(D1):D759D65.

6. Schnoes AM, Brown SD, Dodevski I, Babbitt PC. Annotation error in public databases: misannotation of molecular function in enzyme superfamilies. PLoS Comput Biol. 2009;5(12):e1000605.

7. Prada CF, Boore JL. Gene annotation errors are common in the mammalian mitochondrial genomes database. BMC Genomics. 2019;20(1):73.

8. McDonnell E, Strasser K, Tsang A. Manual gene Curation and functional annotation. Fungal Genomics. Humana Press, New York, NY: Springer; 2018. p. 185-208.

9. Hosmani PS, Shippy T, Miller S, Benoit JB, Munoz-Torres M, Flores-Gonzalez $M$, et al. A quick guide for student-driven community genome annotation. PLoS Comput Biol. 2019;15(4):e1006682.

10. Denton JF, Lugo-Martinez J, Tucker AE, Schrider DR, Warren WC, Hahn MW. Extensive error in the number of genes inferred from draft genome assemblies. PLoS Comput Biol. 2014;10(12):e1003998.

11. Hirsch CN, Hirsch CD, Brohammer AB, Bowman MJ, Soifer I, Barad O, et al. Draft assembly of elite inbred line $\mathrm{PH} 207$ provides insights into genomic and transcriptome diversity in maize. Plant Cell. 2016;28(11):2700-14.

12. Springer NM, Anderson SN, Andorf CM, Ahern KR, Bai F, Barad O, et al. The maize W22 genome provides a foundation for functional genomics and transposon biology. Nat Genet. 2018;50(9):1282.

13. Jiao Y, Peluso P, Shi J, Liang T, Stitzer MC, Wang B, et al. Improved maize reference genome with single-molecule technologies. Nature. 2017 546(7659):524

14. Ou S, Liu J, Chougule KM, Fungtammasan A, Seetharam A, Stein J, et al. Effect of Sequence Depth and Length in Long-read Assembly of the Maize Inbred NC358. bioRxiv. 2019:858365. https://doi.org/10.1101/858365.

15. Song JM, Guan Z, Hu J, Guo C, Yang Z, Wang S, et al. Eight high-quality genomes reveal pan-genome architecture and ecotype differentiation of Brassica napus. Nat Plants. 2020;6(1):34-45.

16. Gao L, Gonda I, Sun H, Ma Q, Bao K, Tieman DM, et al. The tomato pangenome uncovers new genes and a rare allele regulating fruit flavor. Nat Genet. 2019:51(6):1044-51.

17. Wang B, Tseng E, Regulski M, Clark TA, Hon T, Jiao Y, et al. Unveiling the complexity of the maize transcriptome by single-molecule long-read sequencing. Nat Commun. 2016;7:11708. 
18. Campbell MS, Law M, Holt C, Stein JC, Moghe GD, Hufnagel DE, et al. MAKER-P: a tool kit for the rapid creation, management, and quality control of plant genome annotations. Plant Physiol. 2014;164(2):513-24.

19. Eilbeck K, Moore B, Holt C, Yandell M. Quantitative measures for the management and comparison of annotated genomes. BMC Bioinformatics. 2009;10(1):67.

20. Vilborg A, Steitz JA. Readthrough transcription: how are DoGs made and what do they do? RNA Biol. 2017;14(5):632-6.

21. Altschul SF, Gish W, Miller W, Myers EW, Lipman DJ. Basic local alignment search tool. J Mol Biol. 1990;215(3):403-10.

22. Stelpflug SC, Sekhon RS, Vaillancourt B, Hirsch CN, Buell CR, de Leon N, et al. An expanded maize gene expression atlas based on RNA sequencing and its use to explore root development. Plant Genome. 2016;9(1):1-16.

23. Sekhon RS, Briskine R, Hirsch CN, Myers CL, Springer NM, Buell CR, et al. Maize gene atlas developed by RNA sequencing and comparative evaluation of transcriptomes based on RNA sequencing and microarrays. PLoS One. 2013;8(4):e61005.

24. Andrews S. FastQC: a quality control tool for high throughput sequence data; 2010.

25. Martin M. Cutadapt removes adapter sequences from high-throughput sequencing reads. EMBnet Journal. 2011;17(1):10-2.

26. Dobin A, Davis CA, Schlesinger F, Drenkow J, Zaleski C, Jha S, et al. STAR: ultrafast universal RNA-seq aligner. Bioinformatics. 2013;29(1):15-21.

27. Li H, Handsaker B, Wysoker A, Fennell T, Ruan J, Homer N, et al. The sequence alignment/map format and SAMtools. Bioinformatics. 2009;25(16): 2078-9.

28. Anders S, Pyl PT, Huber W. HTSeq-a Python framework to work with highthroughput sequencing data. Bioinformatics. 2015;31(2):166-9.

29. Wagner GP, Kin K, Lynch VJ. Measurement of mRNA abundance using RNAseq data: RPKM measure is inconsistent among samples. Theory Biosci. 2012;131(4):281-5.

30. Delcher AL, Salzberg SL, Phillippy AM. Using MUMmer to identify similar regions in large sequence sets. Curr Protoc Bioinformatics. 2003;1:10 3. 1-3. 8.

31. Li H. Minimap2: pairwise alignment for nucleotide sequences. Bioinformatics. 2018;34(18):3094-100.

32. Quinlan AR, Hall IM. BEDTools: a flexible suite of utilities for comparing genomic features. Bioinformatics. 2010;26(6):841-2.

33. Love M, Anders S, Huber W. Differential analysis of count data-the DESeq2 package. Genome Biol. 2014;15(550):10.1186.

34. Reyes A, Anders S, Huber W. Inferring differential exon usage in RNA-Seq data with the DEXSeq package; 2013.

\section{Publisher's Note}

Springer Nature remains neutral with regard to jurisdictional claims in published maps and institutional affiliations.

Ready to submit your research? Choose BMC and benefit from:

- fast, convenient online submission

- thorough peer review by experienced researchers in your field

- rapid publication on acceptance

- support for research data, including large and complex data types

- gold Open Access which fosters wider collaboration and increased citations

- maximum visibility for your research: over $100 \mathrm{M}$ website views per year

At $\mathrm{BMC}$, research is always in progress.

Learn more biomedcentral.com/submissions 